\title{
Neurofibromatosis I (NF-I): features on MRI
}

\author{
M Grobbelaar, $M B$ ChB \\ R George, MB BS, DMRD, MD (Radiology), \\ MMed (Radiodiagnosis) \\ S H Theron, MB ChB, MMed \\ (Radiodiagnosis), FCRad \\ A Mapukata, MB ChB \\ S Andronikou, MB BCh, FCRad, FRCR (Lond), \\ PhD \\ Department of Radiology, Tygerberg Hospital and Stellenbosch University
}

Magnetic resonance imaging (MRI) can identify 3 of the 7 criteria that are used to diagnose neurofibromatosis I (NF-I). These criteria are optic pathway gliomas, plexiform neurofibromas and sphenoid hypoplasia. ${ }^{1}$ Other features of NF-I visible on MRI of the brain include myelin vacuolisation, vascular dysplasia, spinal lesions (bony abnormalities as well as cord and nerve root lesions), cerebral gliomas, CNS hamartomas, neurofibromas and generalised or local cerebral atrophy. ${ }^{1,2}$

With regard to optic pathway gliomas, MRI is used to show the extent of the tumour, relation to blood vessels, calcifications, presence of cysts,

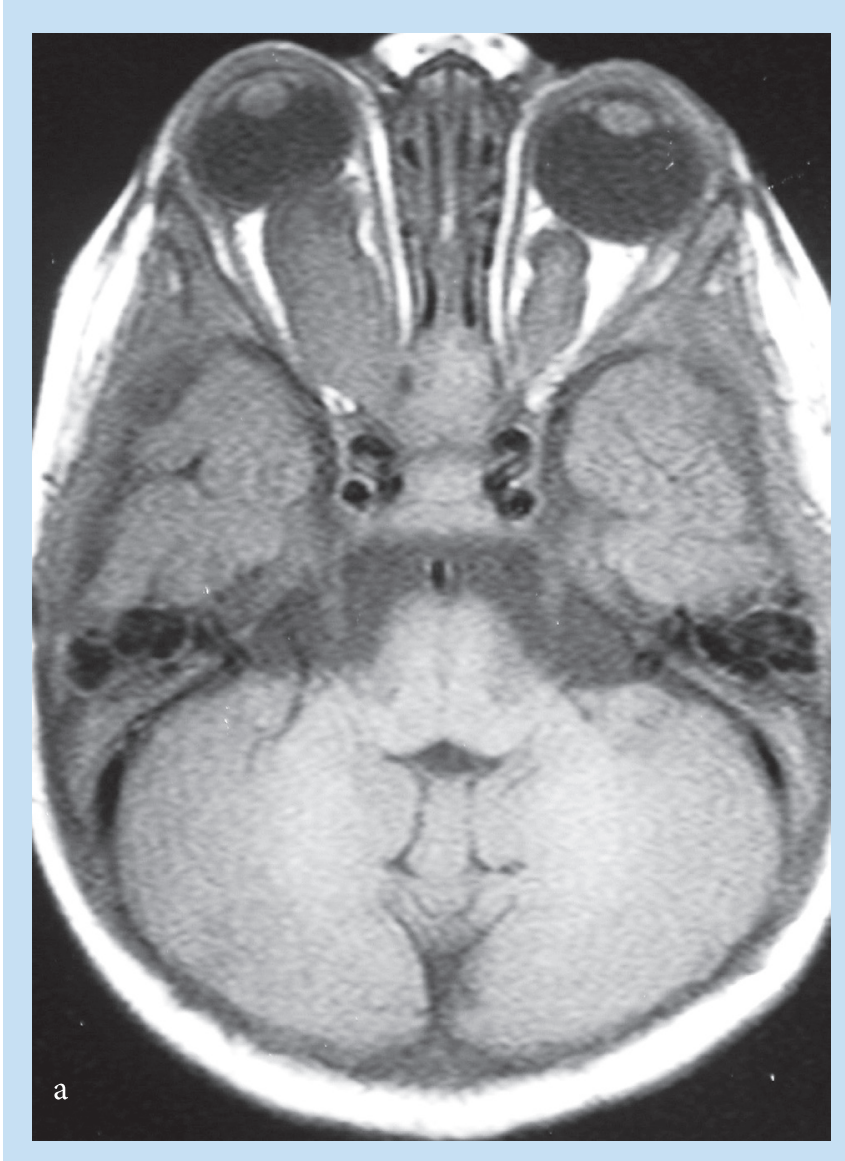

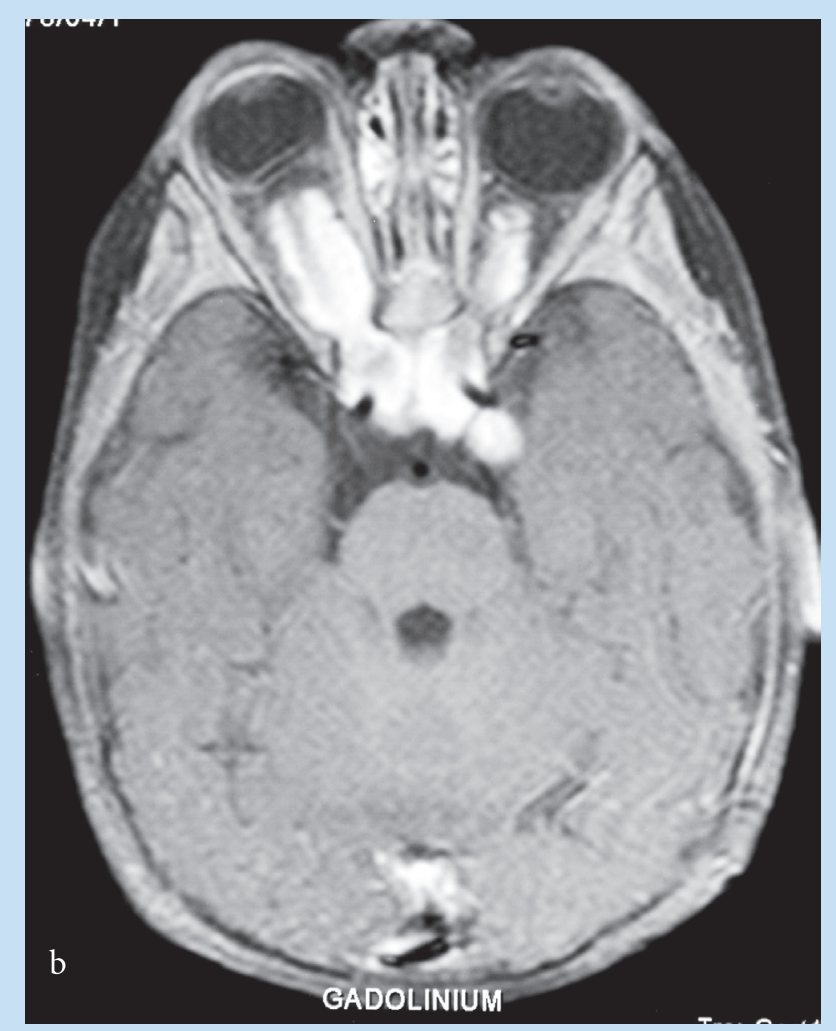

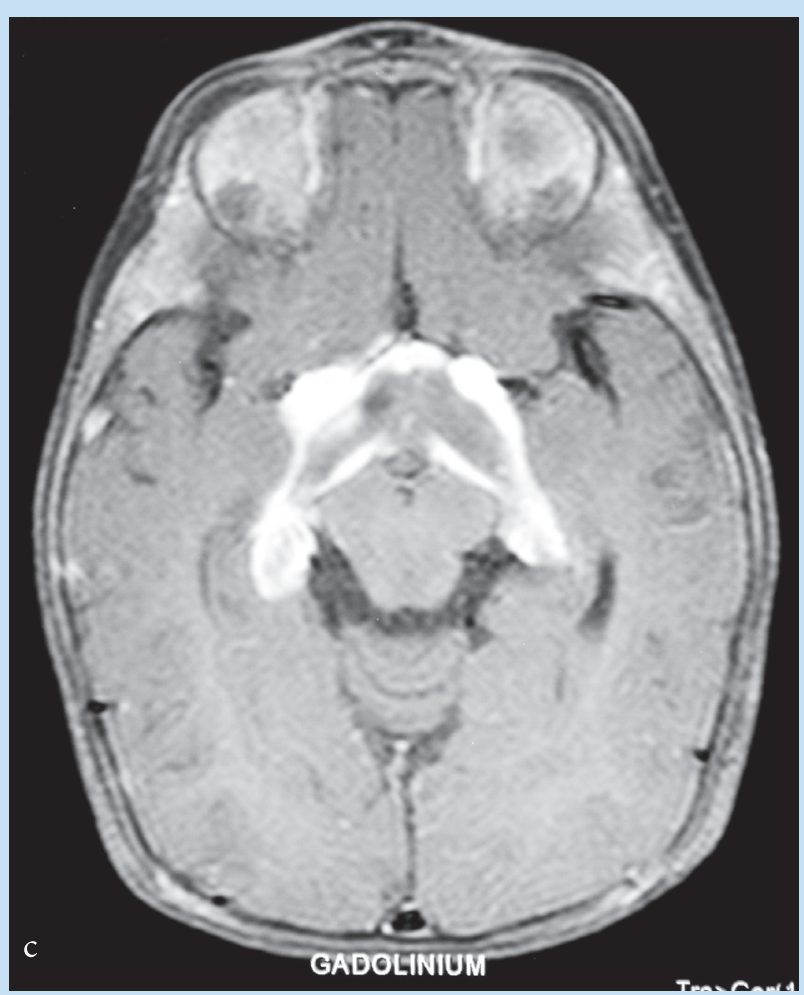

Fig. 1. Bilateral optic pathway gliomas. a. $T 1$ axial image shows bilateral fusiform enlargement of the optic nerves extending through the optic canals. b. T1 post gadolinium with fat suppression image shows diffuse enhancement of the optic nerves. c. T1 post gadolinium with fat suppression image shows extension of the tumour to the chiasm and tracts. 


\section{PICTORIAL INTERLUDE}
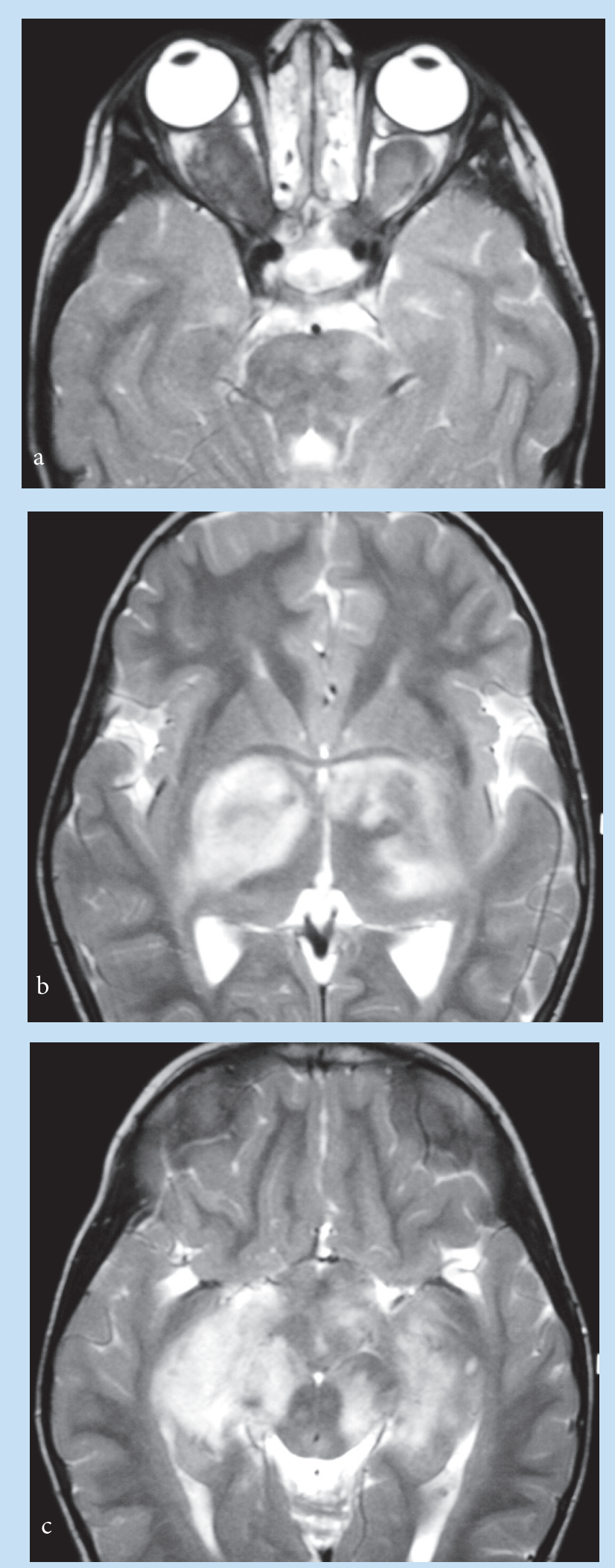

Fig. 2. Bilateral optic pathway gliomas. Sequential axial T2 images. a. Bilateral enlargement of the optic nerves. $b$ and c. Extension of tumour past the optic chiasm along the optic tracts.

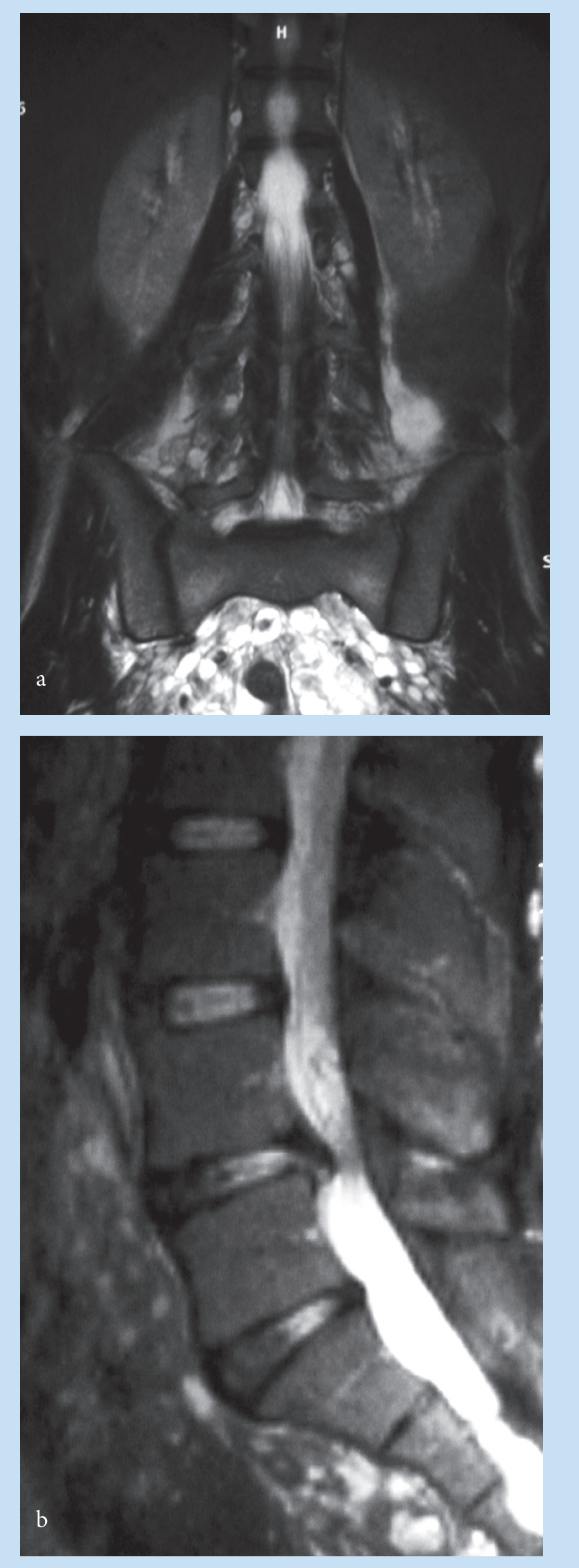




\section{PICTORIAL INTERLUDE}

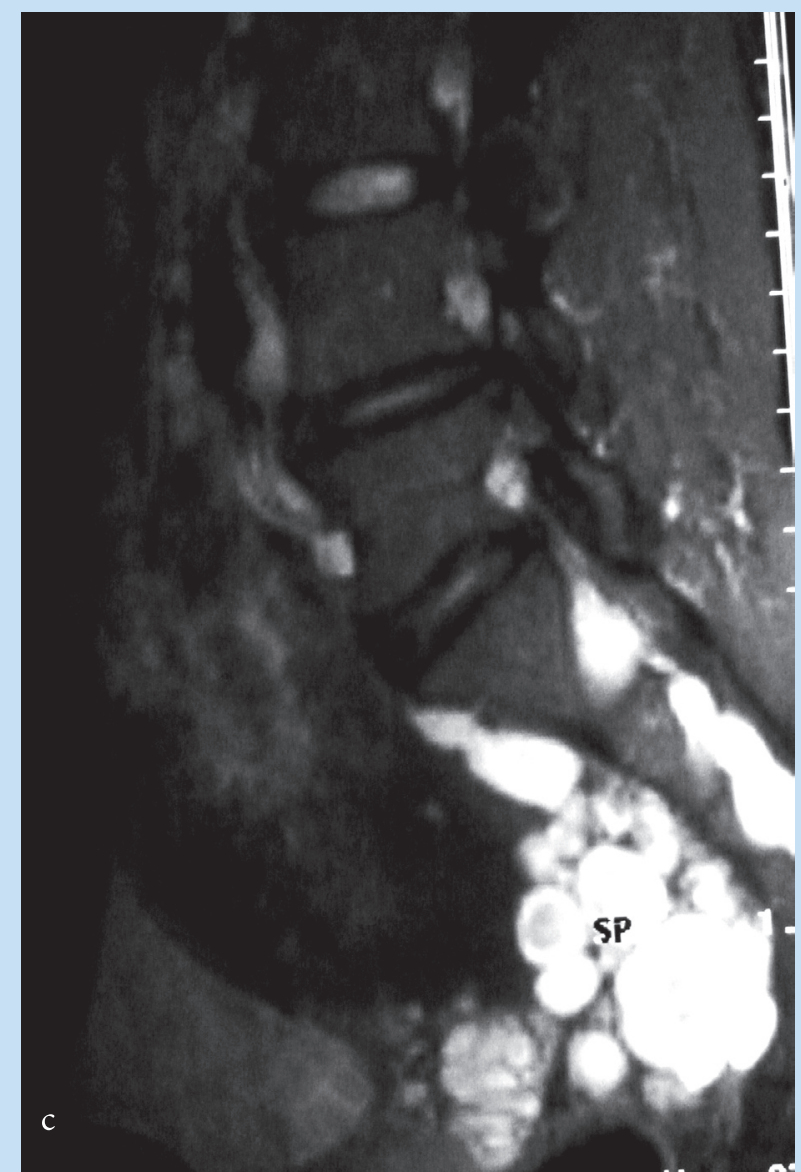

Fig. 3. Plexiform neurofibromata. a. T2 coronal image shows typical target lesions. b. T2 sagittal image showing scalloping of the posterior vertebral bodies secondary to dural ectasia. C. T2 sagittal image with typical target lesions.

peritumoral oedema and enhancement after contrast injection ${ }^{3}$ (Figs 1 and 2). Optic pathway gliomas rarely involve the optic radiations., ${ }^{3,4}$ Specific signs of NF-I in patients with optic pathway gliomas are bilaterality and a fusiform tumour of high signal intensity surrounding a core of lower signal intensity on T2-weighted images. The second sign corresponds to the histopathologic pattern of perineural arachnoidal gliomatosis which is a special feature of orbital gliomas in NF-I. Elongation and downward kinking of the nerves also typify orbital gliomas in these patients. ${ }^{5,6}$

Plexiform neurofibromas are locally aggressive congenital lesions that progress along the nerve of origin. On T2-weighted images the typical 'target sign' is seen where the periphery of the lesions tends to be high signal intensity compared with the low signal intensity of the centre of the lesions (Fig. 3). They may extend intracranially and cause compression and distortion of the brain. They commonly develop in the orbit where they cause impaired ocular movements and exophthalmos. Extension into the cavernous sinus, nasopharynx or pterygomaxillary fissure can occur.

Myelin vacuolisation is present in $75 \%$ of NF-I cases and occurs at specific ages: absent at 0-2 years, increases in number and size at 3-12
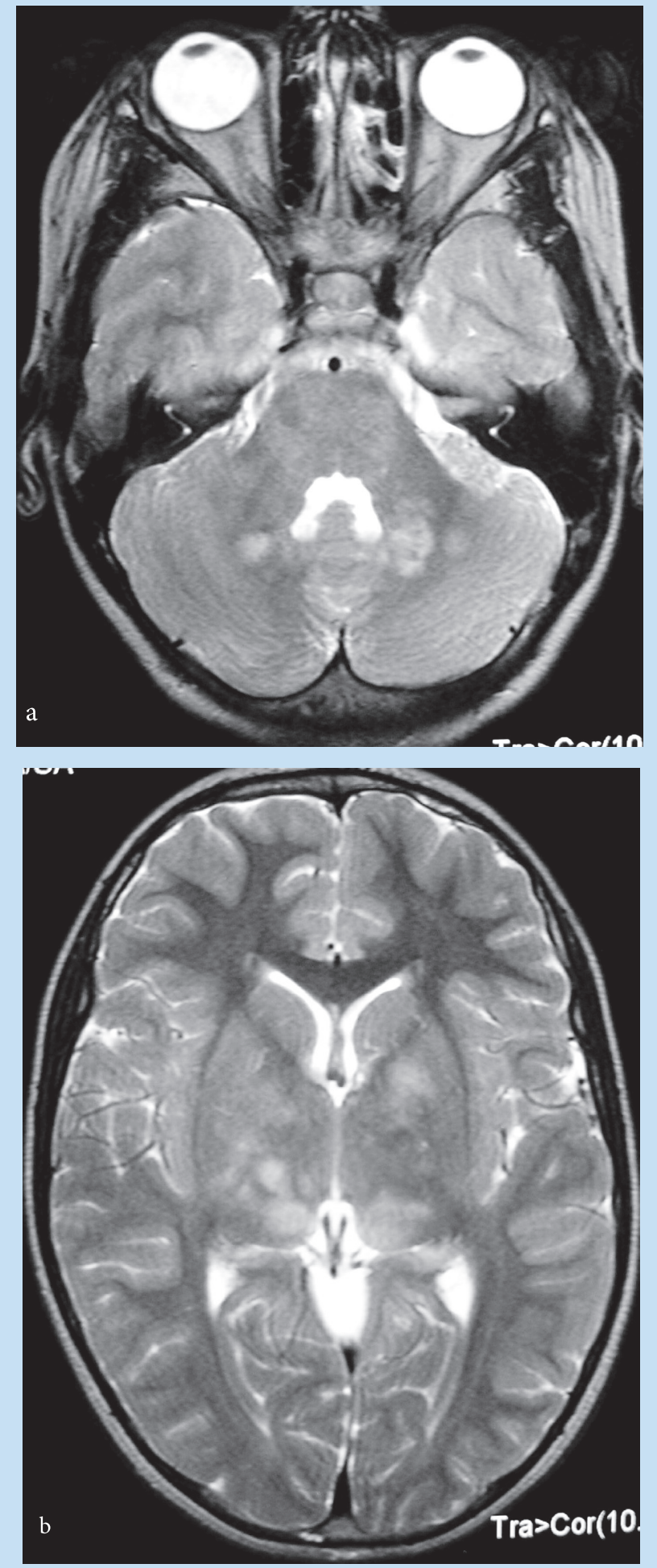

Fig. 4. Foci of myelin vacuolisation in NF-I. a. T2 axial image shows multiple high signal intensities in the cerebellar white matter. $b$. T2 axial image shows multiple high signal intensities in the internal capsule.

years, decreases in number and size at 12-20 years and disappears after 20 years of age. ${ }^{1}$ The foci of abnormal increased signal intensity are seen 


\section{PICTORIAL INTERLUDE}

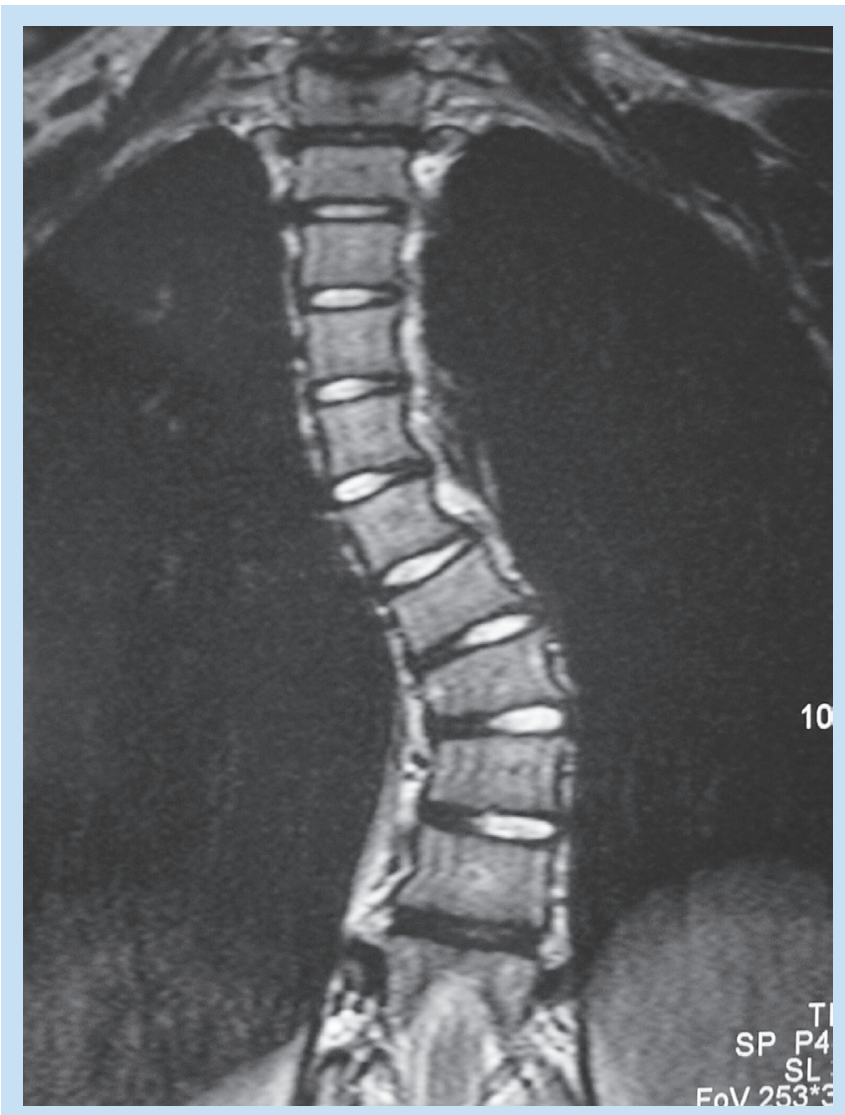

Fig. 5: Scoliosis in NF-I. T1 coronal image shows a typical short-curve thoracic scoliosis characteristic of NF-I.

in the pons, cerebellar white matter, internal capsule and splenium of the corpus callosum on T2-weighted images. The lesions are typically multiple with no mass effect, no vasogenic oedema, normal signal intensity on T1-weighted images, and they are not enhanced by IV contrast administration (Fig. 4). Myelin vacuolisation can also occur in the globus pallidus but these foci differ in radiological appearance in that they are slightly hyperintense on T1-weighted images. ${ }^{7}$

Vascular dysplasia results in stenosis or occlusion of the common or internal carotid artery, proximal middle cerebral artery (MCA) or anterior cerebral artery (ACA) (Fig. 5). Cerebral aneurysms and arteriovenous fistulas occur less commonly. ${ }^{\text {? }}$

Scoliosis is the most common skeletal abnormality reported in NF-I (Fig. 6). It is caused by dysplasia of the vertebral bodies, pedicles or transverse processes, but may also be the result of neurofibromata. ${ }^{1,7}$

1. Andronikou S, Wieselthaler N, Kader E. Baby Steps into Paediatric Neuroradiology. 1st ed. Cape Town: SAMA Health and Medical Publishing Group, 2004: 25-29.

2. Sutton D. Textbook of Radiology and Imaging. 7th ed. London: Churchill Livingstone, 2002: 1737-1738 .

3. Kornreich L, Blaser S, Schwarz M, et al. Optic pathway glioma: correlation of imaging findings with the presence of neurofibromatosis. Am J Neuroradiol 2001; 22: 1963-1969.

4. Grant TL, Brodsky MC, Phillips PC, et al. Optic radiation involvement in optic pathway gliomas in neurofibromatosis. Am J Ophthalmol 2004; 137: 407-414.

5. Brodsky MC. The "pseudo-CSF" signal of orbital optic glioma on magnetic resonance imaging: a signature of neurofibromatosis. Survey of Ophthalmology 1993; 38(2): 213-218.
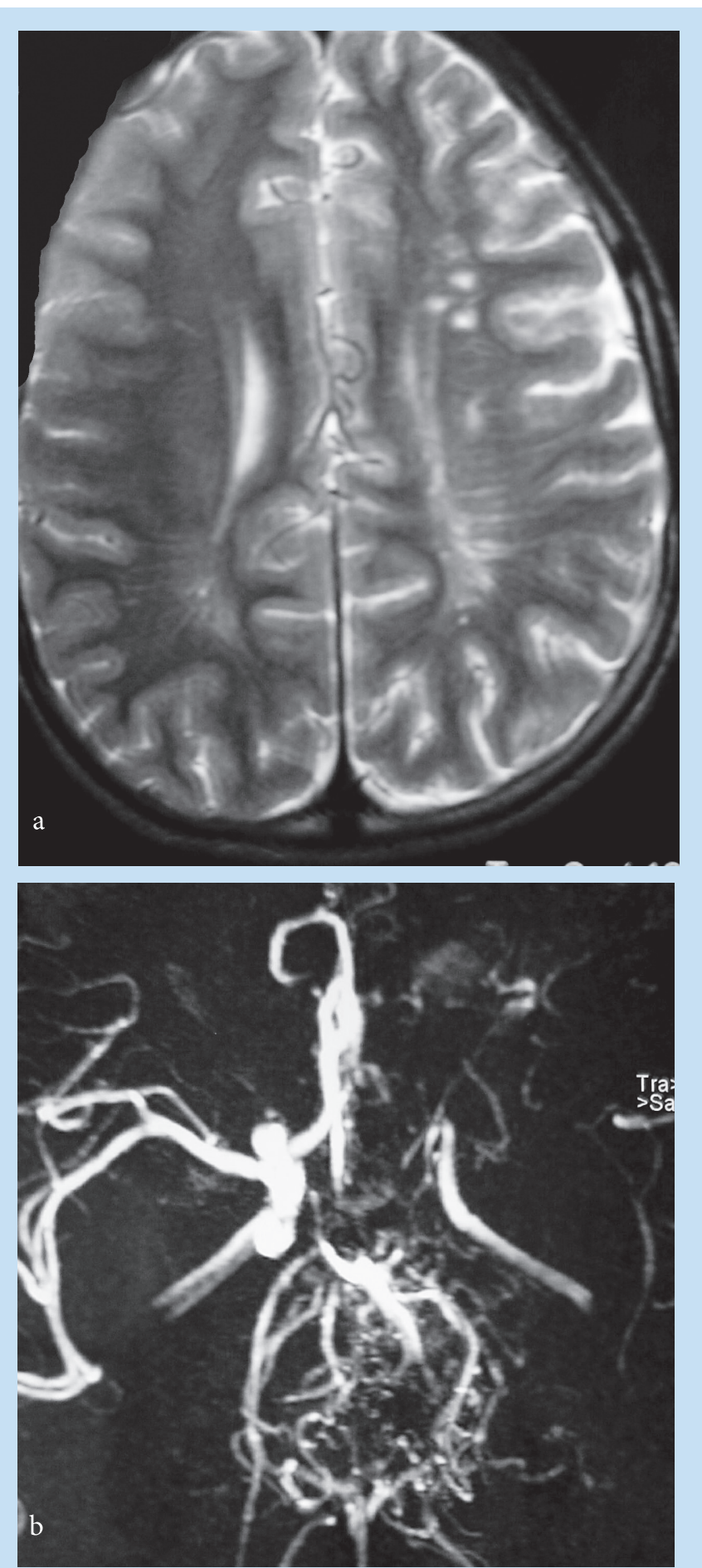

Fig. 6: Vascular dysplasia in NF-I. a. T2 axial image shows asymmetrical atrophic changes of the left hemisphere as a result of vascular compromise. b. MR angiography of the brain shows occlusion of the left proximal ACA and MCA with multiple collateral vessels - moya-moya type pattern.

6. Imes RK, Hoyt WF. Magnetic resonance imaging signs of optic nerve gliomas in neurofibromatosis 1. Am J Ophthalmol 1991; 111: 729-734.

7. Barkovich AJ. Pediatric Neuroimaging. 3rd ed. New York: Lippincott Williams \& Wilkens, 2000: 390-398. 